\title{
Restoration of the femoral head after collapse in osteoarthrosis
}

\author{
G. O. STOREY AND J. W. LANDELLS
}

Hackney Hospital, London E.9, and Department of Morbid Anatomy, Institute of Pathology, The London Hospital

It has often been said that osteoarthrosis of the hip is a relatively static condition, the radiological appearance remaining unchanged over a number of years. It is now recognized, however, that in some cases destructive changes occur (Isdale, 1962; Storey, 1968); some of these may be the result of avascular necrosis. In a proportion of these patients, 'healing' may bring about re-formation of the femoral head and reappearance of the radiological 'joint space'. In the following report this course was observed clinically and radiologically during the last years of the patient's life and correlated with the findings at the postmortem examination.

\section{Case Report}

The patient was first seen in 1966, when he was 76 years old. He gave a history that 8 years previously, in 1958, he had fallen on his right hip. He attended the Casualty Department, where a radiograph (Fig. 1) was thought to be normal. The pain in the hips disappeared, but returned in 1964, especially on walking, about 18 months before his attendance. He also noticed that his leg had become shorter.

\section{Examination}

The shortening of the right lower limb was confirmed (1 in.; $2.5 \mathrm{~cm}$.) with limitation of the movements of the hip. On December 12, 1966, a radiograph (Fig. 2) showed the presence of cystic and sclerotic changes in the head of the right femur with some collapse. The patient had suffered from dysphagia due to benign oesophageal stricture, and the hip changes could be seen to have been present in the radiographs taken for a barium meal in 1965.

\section{Treatment}

Because of his age and because pain was not a marked feature conservative treatment of the osteoarthrosis was decided upon. He was given physiotherapy, indomethacin $25 \mathrm{mg}$. twice daily, and paracetamol as necessary. The condition of the hip remained stationary with no great change in the radiological appearance.

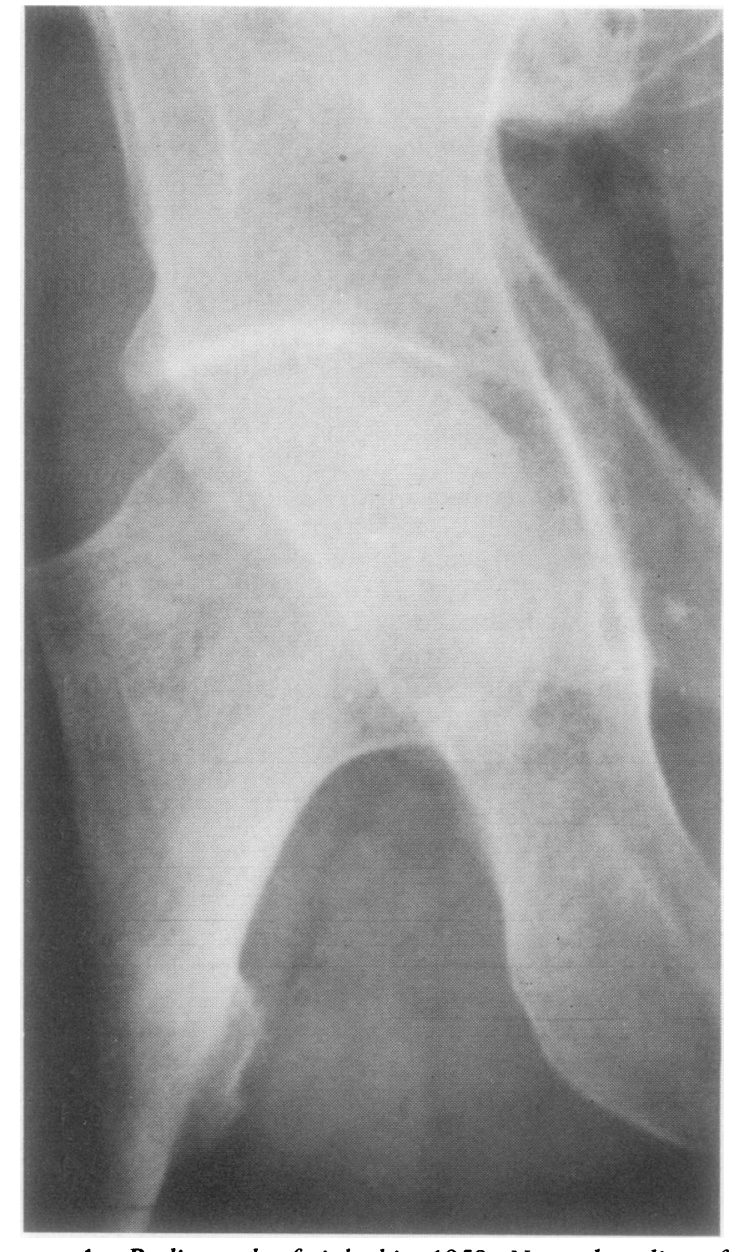

FIG. 1 Radiograph of right hip, 1958. Normal outline of femoral head.

\section{Progress}

The dysphagia continued to be troublesome, and on September 5, 1969, a further radiological examination in the course of barium studies showed that the outline of the head of the femur had now become more defined, 


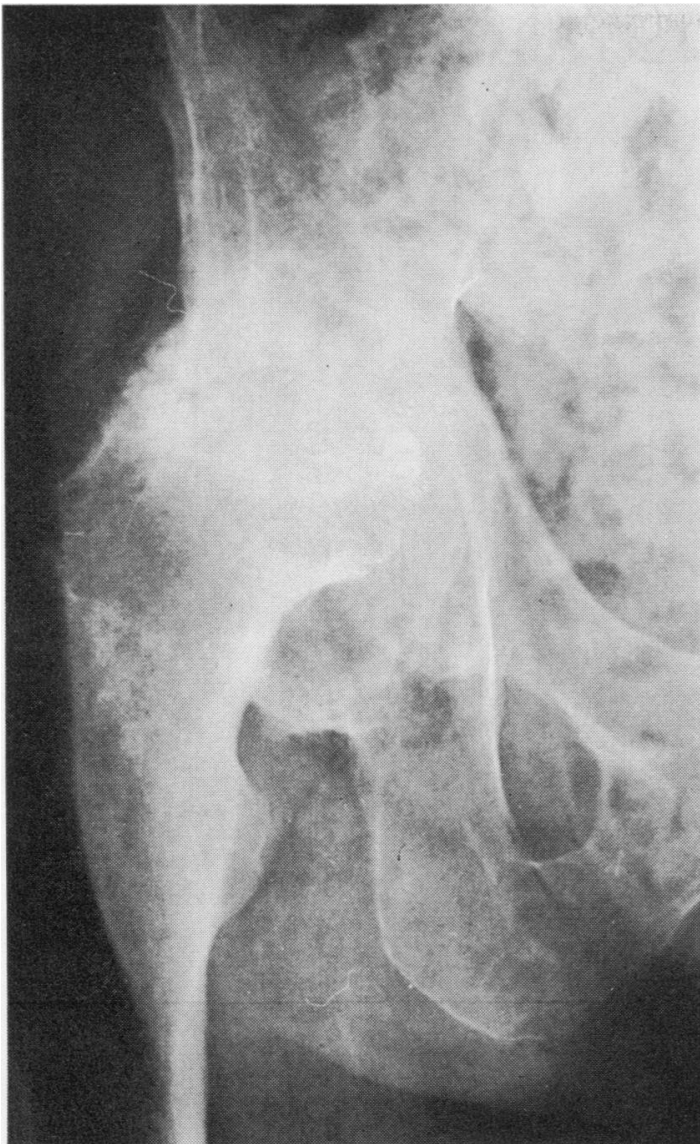

though altered in shape, and that a joint-space had reappeared (Fig. 3).
FIG. 2 Radiograph of right hip, 1966. Sclerotic changes in upper part of head and acetabulum, with obliteration of 'joint space' and displacement of the head upwards relative to acetabulum.

\section{Termination}

The patient had become mentally confused, and he died on September 16, 1969, after 3 weeks in hospital. Death was due to cerebral glioma.

\section{Pathological examination}

The right hip-joint, together with the adjacent part of the bony pelvis and the upper end of the femur, including the lesser trochanter, was excised unopened at autopsy (Dr. J. A. U. Morgan). The whole was divided along a coronal plane in the line of the neck of the femur, fixed, photographed, and examined by ultra-violet light. Blocks were taken for histology from the mid-plane, the anterior surface of the femoral head, three other areas of the joint surfaces, and two loose bodies.

\section{Gross appearance (Figs 4-6, overleaf)}

The head was of nearly normal contour, a little flattened on the upper surface and a little larger than normal $(5 \cdot 5 \mathrm{~cm}$. diameter); anteriorly, the cartilage was rounded, whitish, greyer locally where thinner, with a broad grooved osteophyte on the lower border, and several small nodular osteophytes in the broad sulcus surrounding the articular surface. There was a larger osteochondral nodule below,

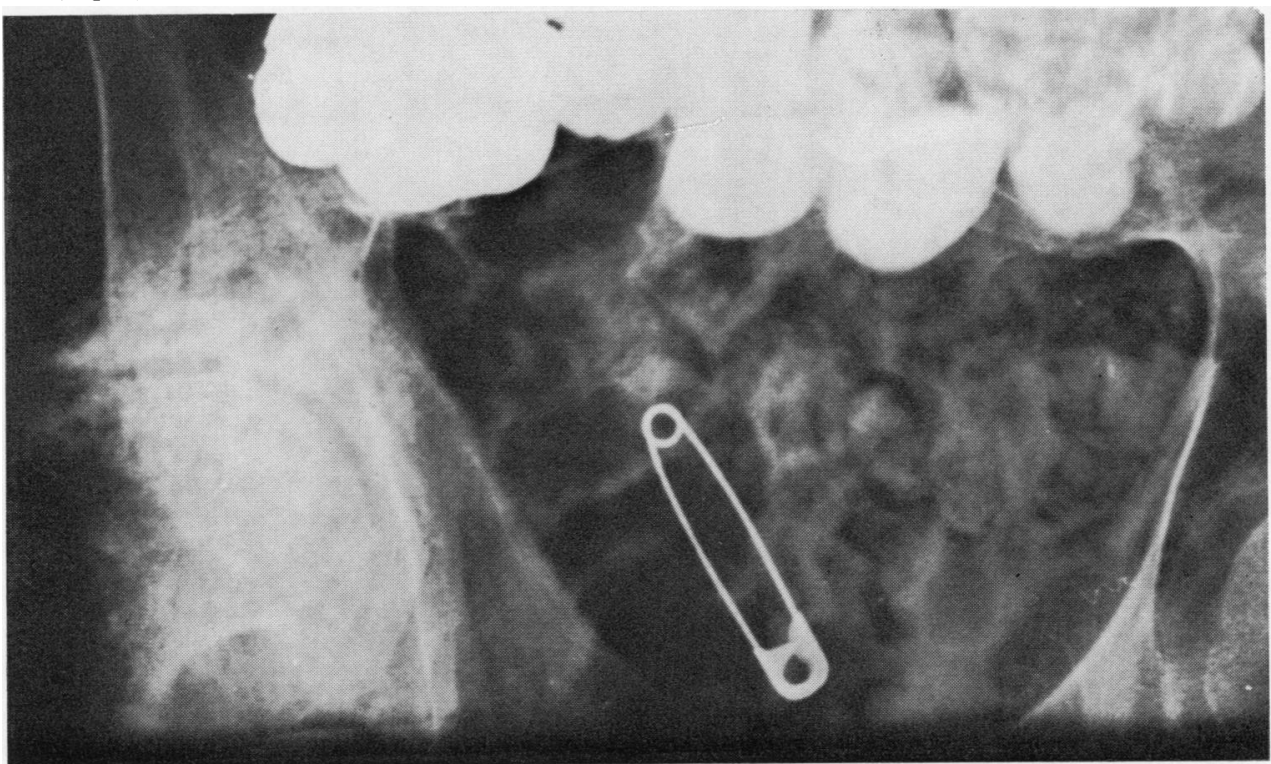

FIG. 3 Radiograph of right hip, 1962. The displacement of the head is still visible, but there is now a clear joint-space and the outline of the head, though showing sclerotic and cystic changes, is very much clearer. 


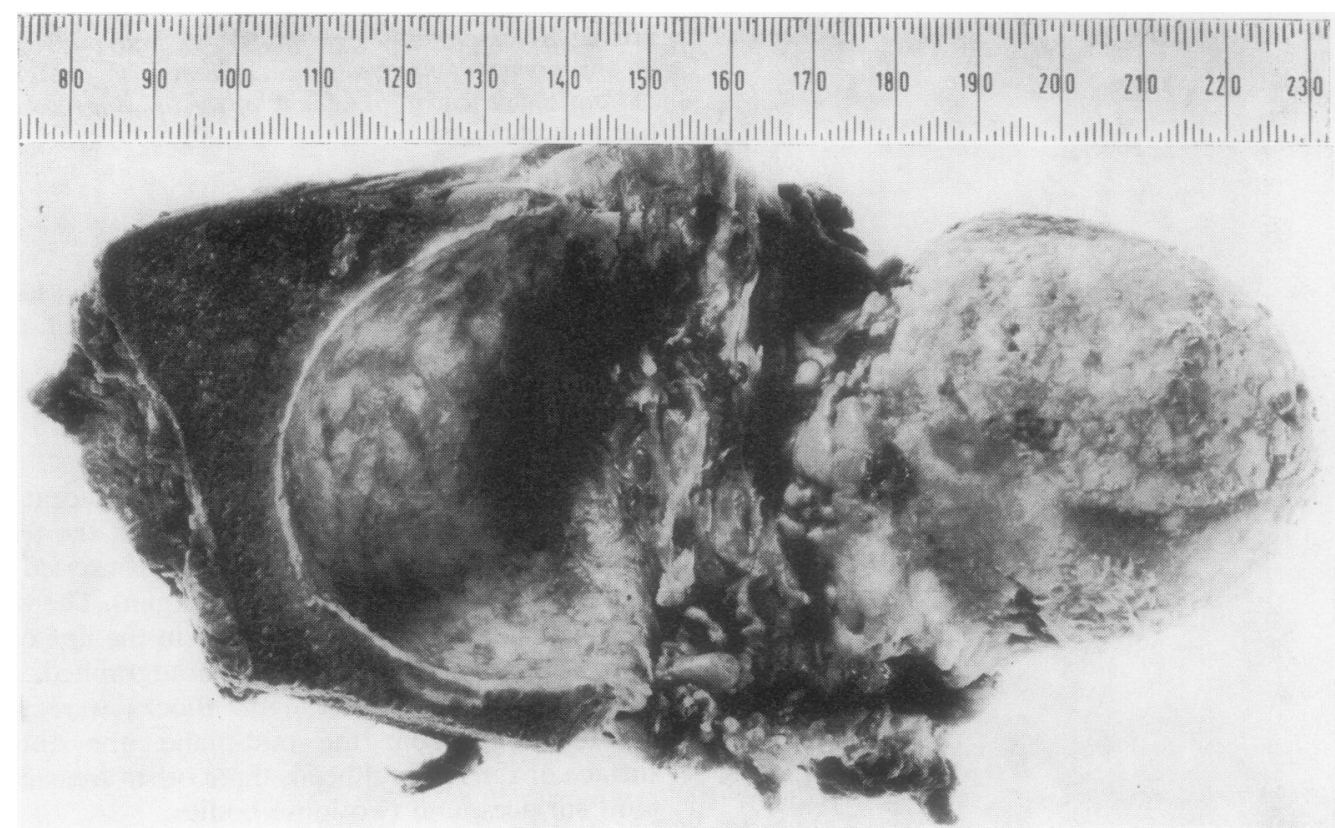

FIG. 4 Anterior view of hip at autopsy. Outline of bone not greatly deformed; nodular osteophytes at junction with neck and synovial villi.

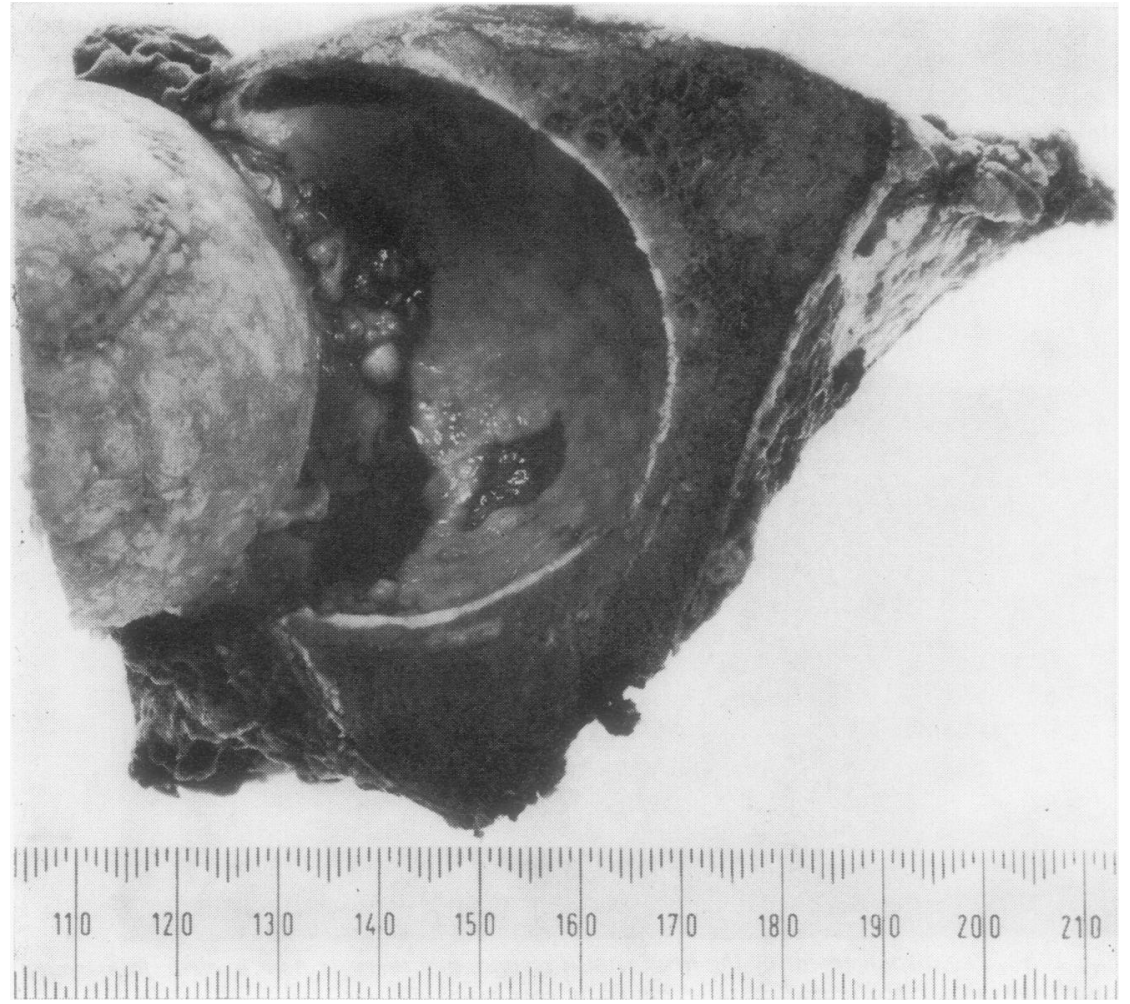

FIG. 5 Posterior view of hip at autopsy. A narrow curved band rather above the middle is the only remaining visible relic of $\bigcirc$ the original articular cartilage. Osteophytic cartilage below this and fibrocartilage above it have restored a remarkably smooth outline to the posterior part of the head. Synovial chondromata and villi and $a$ defect in the acetabular cartilage are also present. 


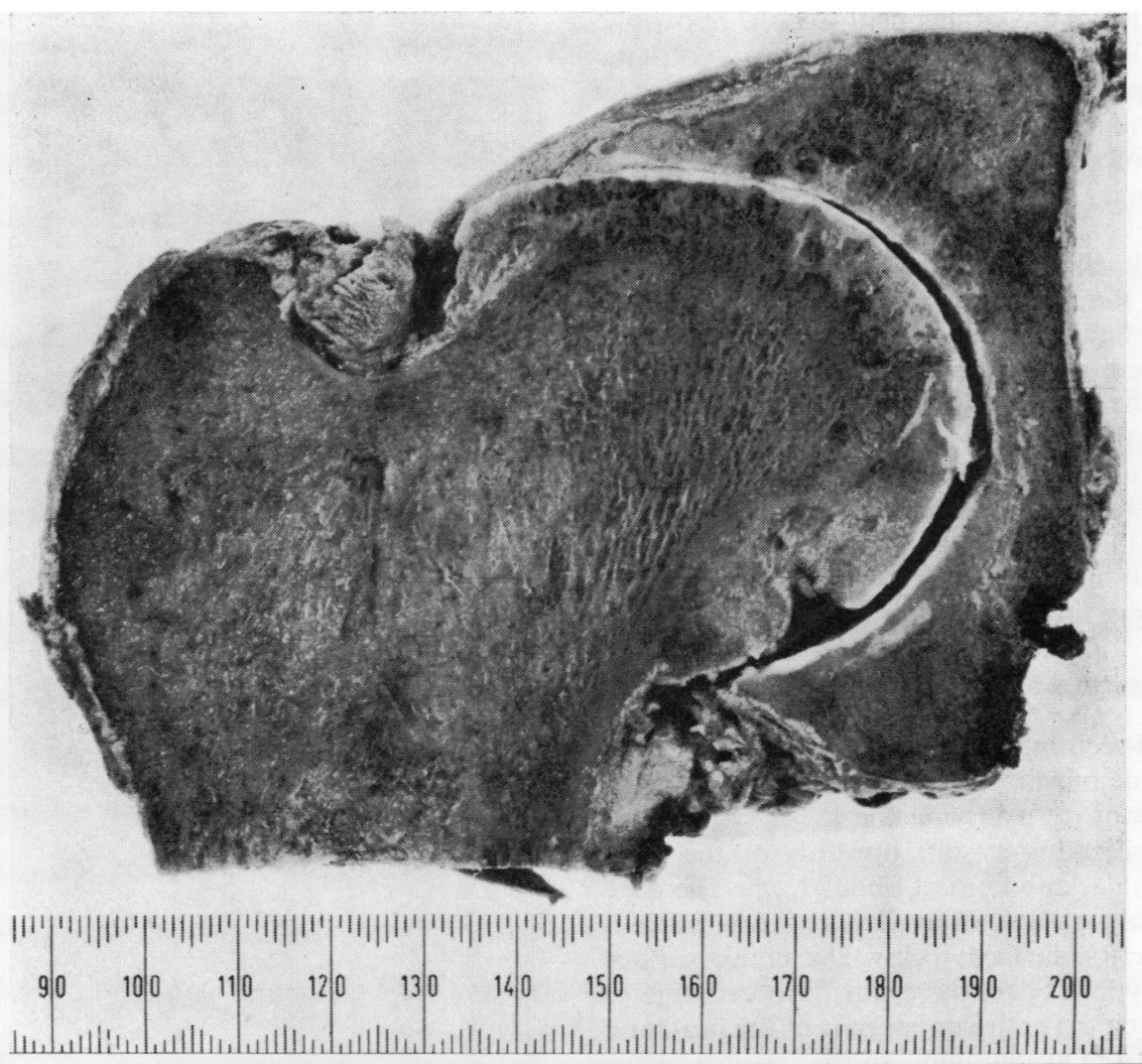

$6 a$

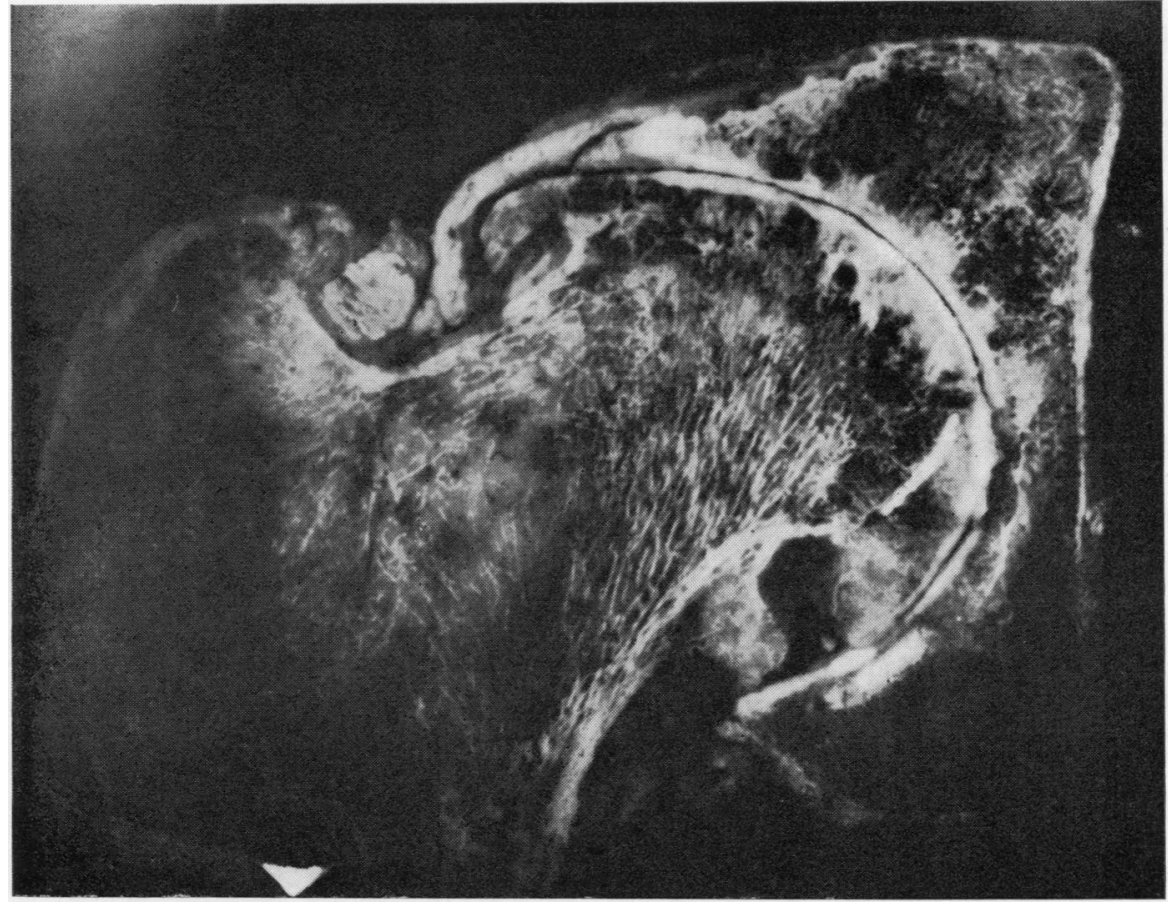

$6 b$

FIG. 6 Mid-section (a) by ordinary light, (b) by ultra-violet autofluorescence. The original outline of the neck and lower articular cartilage of the head can be made out clearly, with osteophytes below and sclerotic bone above. 
which was free of any attachment to bone or capsule but was held in place by other nodules until the joint was opened and the osteophyte worked out of its cavity, the irregularities of its shape corresponding to that of neighbouring nodules; its description as a loose body was correct in the sense that it had no vascular or other attachment, but it was not freely mobile inside the joint. The surfa $e$ of the head facing the acetabulum was slightly rougher in texture, and had a local area of exposed bone a little above the position of the fovea, but was in general rounded. The acetabular cartilage, apart from a sharply-cut defect down to bone near the lower border, was also wholly covered with a sheet of new, slightly rough blue-grey cartilage, much less slippery than normal but equally smoothly contoured. A second 'loose' body was locked in position between the lower edge of the head and a synovial osteochondroma; some smaller synovial nodules and villi were also present.

In the mid-section, the outline of the original head could be made out in the lower half (Fig. 6a) with a smooth osteophyte overlying the lower part of the bone and indicating a shift upwards of the head relative to the acetabulum of about $1 \mathrm{~cm}$. The edge of the original cartilage survived as a narrow crescent on the posterior surface; below the entire surface was osteophytic; above, there was fibro-cartilage of repair covering the entire upper part of the head and the whole of the acetabulum. In the lower part the doubled cartilage (new inside the old) could be clearly seen.

These changes were verified by examination under ultra-violet light, which provides a clear distinction between hyaline articular cartilage (greyish-white autofluorescence) and fibrocartilage which, whether it is formed in repair of defects in the original surface or as a surface of an osteophyte, has a strong blue autofluorescence. The calcified zone between normal cartilage and bone stands out as a bright chalky white, and bone trabeculae are much more distinctly shown than by ordinary light. Unfortunately these colours do not reproduce satisfactorily in black and white for publication, but something of the changes can be made out in Fig. 6(b).

\section{HISTOLOGICAL EXAMINATION (Fig. 7)}

This confirmed that none of the surface cartilage had the structure of normal hyaline or even of fibrillated osteoarthrotic cartilage, and that the normal articular lamella was missing. None of the bone of the head was now necrotic-indeed much of it had undergone the usual reinforcement of an osteoarthrotic head. There was a core of dead bone, consistent with origin in the head, in one only of the loose bodies. The synovium showed the usual

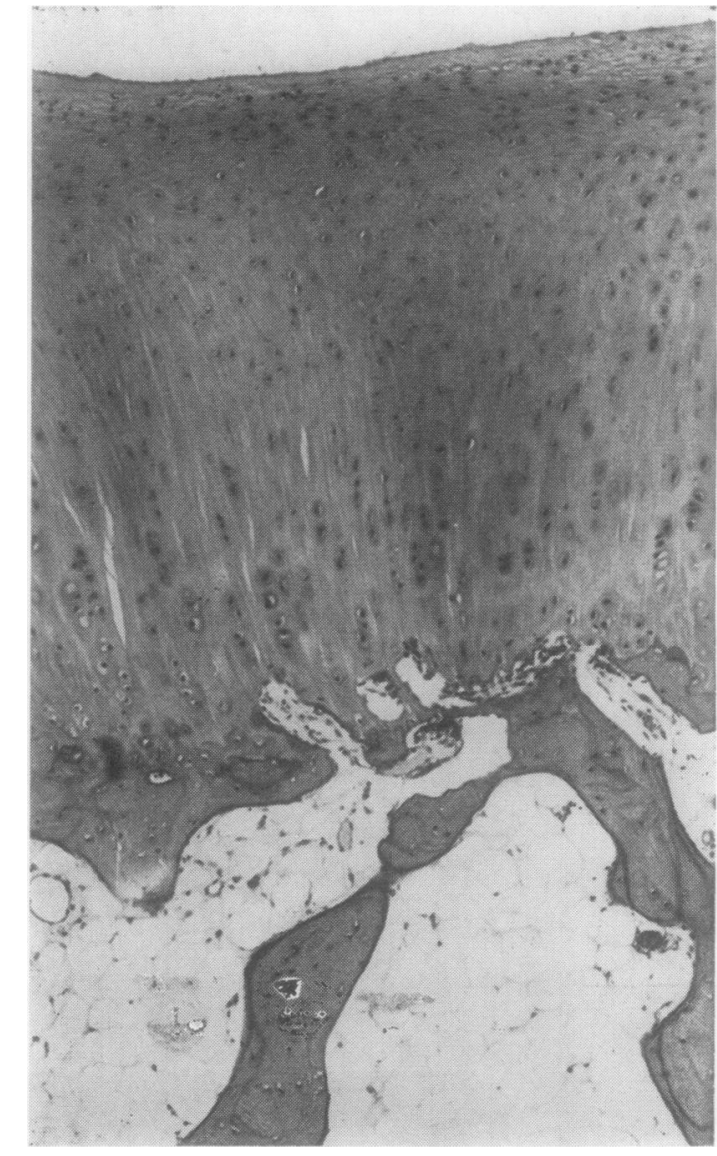

FIG. 7 Photomicrograph of new surface cartilage of joint: The matrix is fibrillar and not hyaline, the cells often lying singly instead of in the groups of normal articular hyaline cartilage. The underlying bony sheet shows the variable activity of a recently formed piece of bone, and not the stable inactive sheet and supporting struts of a normal articular lamella. Haematoxylin and eosin, $\times 30$.

villous proliferation and debris of osteoarthrosis, but was without rheumatoid features.

Evidence that a large part of the head of the femur was in fact destroyed:

(1) The $x$-ray photographs (Figs 1 to 3 during life, and Fig. 8 of the central slice of the excised bone) show that the internal architecture of the trabeculae is distorted; there is no articular bony plate, and the epiphyseal line is not traceable.

(2) The present configuration of the head is explicable only by the disappearance of the upper part of the bone, allowing the original line of the lower border to move up and an osteophyte about $1 \mathrm{~cm}$. thick to form below it. This osteophytic restoration of the lower part of the bearing surface is commonly seen in osteoarthrotic hips, but is usually associated with gross defects in the upper surface. 


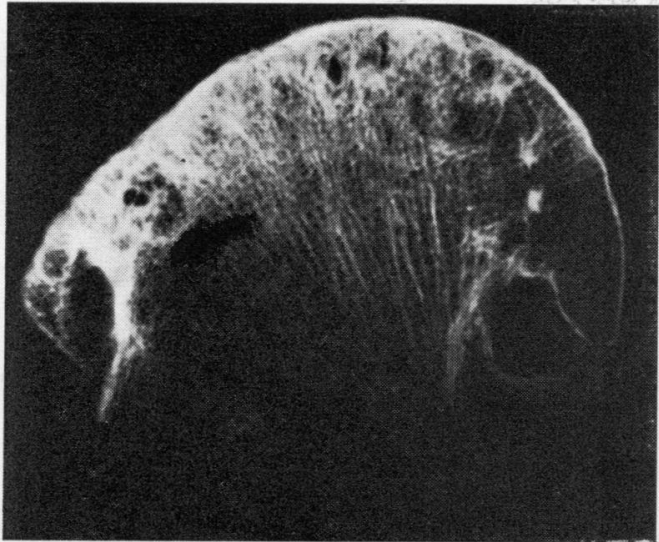

FIG. 8 Radiograph of anterior part of head, showing loss of normal architecture and cystic changes.

(3) The histological structure of the new upper part of the bone is not that of normal or of osteoarthrotic articular cartilage, but resembles that seen in repair by the orthopaedic technique of arthroplasty. While part of the success of the repair in this patient may be explained by the relative lack of vigorous use of the joint in an ill, elderly man, the result in this hip is sufficiently uncommon in osteoarthrosis to suggest that there were other factors in stabilization, in particular the large strong anterior osteophyte which may have helped to prevent partial dislocation and fracture of the damaged bone in the weak early stages of repair.

It is noteworthy that, although fibro-cartilage had reformed in this patient, no improvement in his symptoms could be detected; in other cases too in which there has been a reappearance of joint space radiologically, this has not been associated with lessening of pain.

\section{Discussion}

Although it was possible to follow the clinical course in this patient, it remains uncertain whether the initial event was avascular necrosis precipitated by trauma or not; but the subsequent events were those of uncomplicated osteoarthrosis and there was no evidence of avascular necrosis at autopsy.

The occurrence of this degree of healing in osteoarthrosis as a result of unaided natural forces is very unusual. Harrison, Schajowicz, and Trueta (1953) said that it did occur, and believed that as a result of the destructive process fibrous marrow might reach the surface of the bone and there differentiate into fibrocartilage. Tufts of fibrocartilage arising in this way usually remain discrete, but occasionally become confluent and resurface the bone. Jacobs (1960) illustrated one such outgrowth. Merle d'Aubigny, Postel, Mazabraud, Massias, and Gueguen (1965) considered that small areas of avascular necrosis could be replaced by bone, and repair has been reported after several surgical procedures. Restitution of bone pattern with healing of cysts and improvement of bone outline, as well as a radiological increase in joint space, has been reported after McMurray's osteotomy (Harris and Kirwan, 1964). Adam and Spence (1958) reported this in 38 out of 55 cases. Robins and Piggot (1960), however, believed that the increase in joint space was only occasionally due to the regeneration of articular cartilage and that this radiological appearance was mainly the result of the altered position of the femoral head. The restoration of a surface of normal contour and tolerably smooth fibrocartilage is the usual end-result of the operation of cuparthroplasty.

The reasons for the infrequence of this repair in the natural course of the disease are complex. Formation of even fibrocartilage is a slow process, and requires a steady position of the head in the acetabulum for some months if the new sheet is not to be broken up by a shift of the pressure areas on either side of the joint. The stability of the joint in this patient was secured probably in the main by the two large osteophytes, and reinforced somewhat paradoxically by the complex interlocked synovial osteocartilaginous 'large bodies', both of which limited the range of movement and prevented the weight of the body from compressing the head into the acetabulum; in the usual late osteoarthrotic head, evidence of frequent shifts of pressure and malignment is provided by the common deep groove over the upper part of the junction between the neck and the head, and by microfractures in the bearing surface, even when gross destruction of the surface and large cysts in the substance of the head are not present. Where, however, stability is provided, the extremely vigorous ossification and formation of fibrous tissue and fibrocartilage (though not hyaline cartilage) which is present in every osteoarthrotic joint is fully capable in a year or two of producing and maintaining a bearing surface with sufficient elasticity to serve the patient for many years, though with nothing like the friction-free character of the original hyaline cartilage. Secondly, the vascularity of the healing surface must be preserved at least as far as the relatively low requirements of fibrocartilage are satisfied - by the weight being taken off it to some extent; in the unmodified osteoarthrotic surface sprouting capillaries are obliterated or rubbed away by the pressure and movement of the eburnated and irregular parts of the surface. It is not of great moment how the stability is achieved-by taking the weight on another piece of the femoral neck in McMurray's operation, by taking it on osteophytes as in this patient and in a small number of others with less complete or effective restoration, 
or by the insertion of a smooth metal cup to equalize and spread over the pressure on the bearing surface while healing occurs; in all cases in which this is done effectively the femoral head will re-clothe itself.

\section{Summary}

A case is described in which a smoothly-rounded femoral head was reformed in the natural process of repair, after destruction by osteoarthrosis.

\section{References}

Adam, A., AND Spence, A. J. (1958) J. Bone Jt Surg., 40B, 219 (Intertrochanteric osteotomy for osteoarthritis of the hip. A review of 58 operations).

HARRIS, N. H., AND KIRWAN, E. (1964) Ibid., 46B, 477 (Results of osteotomy for early primary osteoarthritis of the hip).

Harrison, M. H. M., Schajowicz, F., AND Trueta, J. (1953) Ibid., 35B, 598 (Osteoarthritis of the hip: a study of the nature and evolution of the disease).

IsDale, I. C. (1962) Ann. rheum. Dis., 21, 23 (Femoral head destruction in rheumatoid arthritis and osteoarthritis).

JACOBS, J. H. (1960) Ann. phys. Med., 5, 318 (Osteoarthritis of the hip-joint).

Merle d'Aubigny, R., Postel, M., Mazabraud, A., Massias, P., and Gueguen, J. (1965) J. Bone Jt Surg., 47B, 612 (Idiopathic necrosis of the femoral head in adults).

Robins, R. H. C., AND PIGGOT, J. (1960) Ibid., 42B, 481 (McMurray osteotomy, with a note on the 'regeneration' of articular cartilage).

Storey, G. O. (1968) Proc. roy. Soc. Med., 61, 961 (Bone necrosis in joint disease). 